\title{
Special Issue: International Conference on Applied System Innovation (ICASI 2016)
}

\author{
Sheng-Joue Young ${ }^{1} \cdot$ Teen-Hang Meen ${ }^{1} \cdot$ Ajit Khosla $^{2} \cdot$ B. Michel $^{3}$
}

Published online: 3 July 2017

(C) Springer-Verlag GmbH Germany 2017

This special issue presents 37 papers selected from the International Conference on Applied System Innovation (ICASI 2016) held in May 26-30, 2016, at Okinawa, Japan. ICASI 2016 provides a unified communication platform for researchers in a wide area of topics, including information technology, innovation design, communication science and engineering, industrial design, creative design, applied mathematics, computer science, design theory, cultural and creative research, electrical and electronic engineering, mechanical and automation engineering, green technology and architecture engineering, material science, and other related fields. Moreover, this conference enables interdisciplinary collaborations between science and engineering technologists in the academic and industrial fields, as well as networking fields internationally.

Furthermore, this special issue covers a wide range of fundamental studies, industrial practices, and engineering innovations by applying knowledge on microsystems. All of the articles underwent rigorous peer reviews conducted by Microsystem Technologies. The acceptance of this special issue was based on the reviewers' recommendations,

Sheng-Joue Young

shengjoueyoung@gmail.com

Teen-Hang Meen

thmeen@nfu.edu.tw

Ajit Khosla

khosla@gmail.com

B. Michel

bernd.michel@coinn.de

1 Department of Electronic Engineering, National Formosa University, Yunlin 632, Taiwan

2 Faculty of Engineering, Yamagata University, 4-3-16 Jonan, Yonezawa, Yamagata 992-8510, Japan

3 Fraunhofer Micro Materials Center Berlin, Berlin, Germany mandatory revisions, and final examinations, and conducted by the guest editors. It is expected to be a key issue for Microsystem Technologies.

We would like to express our deepest gratitude to the reviewers for taking the time to comment on the papers. Moreover, special thanks are given to the Editor-in-Chief, Prof. Bernd Michel, for his kind support to the publication of this issue.

Finally, we would also like to thank the editorial team for their work to make this special issue a success.

We look forward to your participation in future special issues of Applied System Innovation.

Sincerely,

Guest editors

Prof. Sheng-Joue Young<smiles>C=CCCCCCC(C)C</smiles>

Prof. Teen-Hang Meen

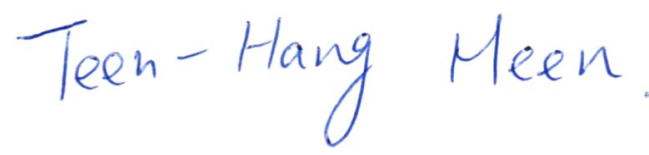

Prof. Ajit Khosla

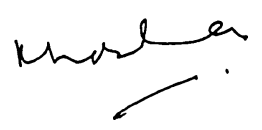

Prof. B. Michel (Editor-in-Chief) 